\title{
Optical rogue waves and soliton turbulence in nonlinear fibre optics
}

\author{
Genty, G.; Dudley, J. M.; de Sterke, C. M.; Eggleton, B. J.; Bang, Ole
}

Published in:

Proceedings of the Conference on Lasers and Electro-Optics and European Quantum Electronics Conference

Publication date:

2009

Document Version

Publisher's PDF, also known as Version of record

Link back to DTU Orbit

Citation (APA):

Genty, G., Dudley, J. M., de Sterke, C. M., Eggleton, B. J., \& Bang, O. (2009). Optical rogue waves and soliton turbulence in nonlinear fibre optics. In Proceedings of the Conference on Lasers and Electro-Optics and European Quantum Electronics Conference: CLEO Europe - EQEC (pp. oral EF2.1). IEEE.

\section{General rights}

Copyright and moral rights for the publications made accessible in the public portal are retained by the authors and/or other copyright owners and it is a condition of accessing publications that users recognise and abide by the legal requirements associated with these rights.

- Users may download and print one copy of any publication from the public portal for the purpose of private study or research.

- You may not further distribute the material or use it for any profit-making activity or commercial gain

- You may freely distribute the URL identifying the publication in the public portal 


\title{
Optical rogue waves and soliton turbulence in nonlinear fibre optics
}

\author{
G. Genty ${ }^{1}$, J. M. Dudley ${ }^{2}$, C.M. de Sterke ${ }^{3}$, B. J. Eggleton ${ }^{3}$, O. Bang ${ }^{4}$
}

1. Tampere University of Technology,

2. Institut FEMTO-ST, Université de Franche-Comté, 25030 Besançon, France

3 CUDOS ARC Centre of Excellence, School of Physics, University of Sydney, NSW 2006 Australia

4. DTU Fotonik, Technical University of Denmark, DK-2800 Kongens Lyngby, Denmark

\begin{abstract}
We examine optical rogue wave generation in nonlinear fibre propagation in terms of soliton turbulence. We show that higher-order dispersion is sufficient to generate localized rogue soliton structures, and Raman scattering effects are not required.
\end{abstract}




\title{
Optical rogue waves and soliton turbulence in nonlinear fibre optics
}

\author{
G. Genty ${ }^{1}$, J. M. Dudley ${ }^{2}$, C.M. de Sterke ${ }^{3}$, B. J. Eggleton ${ }^{3}$, O. Bang ${ }^{4}$ \\ 1. Department of Physics, Tampere University of Technology, FIN-33101 Tampere, Finland, \\ 2. Institut FEMTO-ST, Université de Franche-Comté, 25030 Besançon, France \\ 3 CUDOS ARC Centre of Excellence, School of Physics, University of Sydney, NSW 2006 Australia \\ 4. DTU Fotonik, Technical University of Denmark, DK-2800 Kongens Lyngby, Denmark
}

There is currently intense research into extreme value fluctuations (optical rogue waves) in long pulse fibre supercontinuum (SC) generation [1]. This interest is motivated by the possibility to obtain important insights into related hydrodynamic instabilities, as well as by potential applications in generating stable SC [2]. Optical rogue waves are generated under conditions where SC broadening develops from initial spontaneous modulation instability. Following the appearance of a noise-driven temporal modulation on the input pulse, a large number of distinct solitons emerge, a small number of which undergo extreme Raman frequency shifts to longer wavelengths. Although this suggests that Raman scattering plays a central role in their formation through inelastic energy exchange [3], here we revisit the dynamics of optical rogue wave formation in terms of the well-known soliton turbulence description in which solitons play the role of statistical attractors in any non-integrable nonlinear Schrödinger equation (NLSE) model [4]. Specifically, we show that Raman scattering is unnecessary for intense rogue pulses to emerge from SC generation, and third-order dispersion on its own is sufficient to stimulate a single large rogue soliton in the long distance limit. An additional novel result is that the rogue soliton undergoes a continuous frequency shift to longer wavelengths with propagation as a result of interaction with the turbulent background, even in the absence of Raman scattering.

Our simulations use the fibre nonlinear Schrödinger equation (NLSE) with only third order dispersion and no Raman response. Nonlinearity $\gamma=0.011 \mathrm{~W}^{-1} \mathrm{~m}^{-1}$ and dispersion coefficients $\beta_{2}=-0.41 \mathrm{ps}^{2} \mathrm{~km}^{-1}$ and $\beta_{3}=0.069 \mathrm{ps}^{3} \mathrm{~km}^{-1}$. SC generation is modelled under long-pulse conditions by numerically solving the NLSE with a $500 \mathrm{~W}$ peak power 10 ps pulse input at $1064 \mathrm{~nm}$, and with periodic boundary conditions. Our results show that the initial modulation instability of the input pulse leads to pulse breakup and decay into multiple solitons which then are subject to multiple collisions and interactions [4]. Fig. 1(a) and (b) show the calculated field spectrograms at selected distances where we clearly see the emergence of one distinct soliton pulse in the time-frequency domain. This soliton emerges from a low amplitude broadband turbulent background, and gains energy from the background with propagation as shown in Fig. 1 (c). Significantly, even though no Raman scattering is involved, we still observe a steady frequency shift to longer wavelengths as shown in Fig. 1(d). For comparison, however, we note that the appearance of an isolated rogue soliton in the presence of Raman scattering occurs at propagation distances approximately 10-20 times shorter.

In an optical context, these results are significant in that they show that rogue wave fluctuations in SC generation can occur under conditions where Raman effects are negligible. In the context of SC generation dynamics, this is a new observation, and suggests that frequency-shifting processes in SC generation may contain intrinsic contributions from non-Raman processes. In a wider context, these results also suggest that extreme-value processes may be universal in all systems governed by perturbed NLSE propagation dynamics.
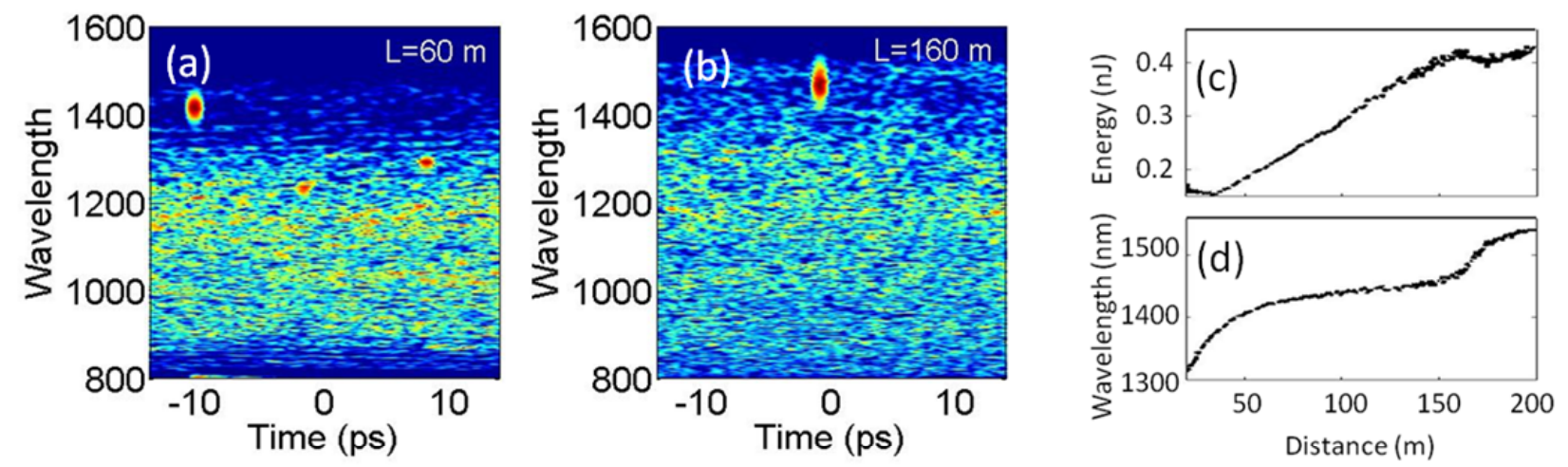

Fig. 1 (a) and (b) spectrogram evolution of SC field at selected distances shown. (c) and (d) Evolution of the energy and the wavelength of the highest intensity soliton as a function of distance.

\section{References}

1. D. R. Solli et al. “Optical Rogue Waves,” Nature 450, 1054 (2007).

2. J. M. Dudley et al., "Harnessing and Control of Optical Rogue Waves in Supercontinuum Generation,” Opt. Express 16, 3644 ( 2008).

3. F. Luan et al. "Energy exchange between colliding solitons in photonic crystal fibers," Opt. Express 14, 9844 (2006).

4. V. E. Zakharov et al., “Soliton Turbulence,” JETP Letters 48, 83 (1988). 